\title{
Application and study on the Hydraulic Switching Power Control
}

\author{
Sumiko SEKI* and Takahiko ITOH* \\ * Department of Electronic and Mechanic Development \\ Yukigaya Seigyo Laboratory \\ 2-12-12-203 Shin-Yokohama, Kohoku-ku, Yokohama, 222-0033 Japan \\ (E-mail: seki_yukigaya@nifty.com, itoh_yukigaya@mbe.nifty.com;)
}

\begin{abstract}
Recently, a technology on the machines under computer control is become established. However huge working power of the hydraulic mechanics is difficult to control with taking efficient power transmission and its precision. Then hydraulic switching power control system is contrived based on analogy between electric circuit and fluid circuit. Hydraulic and electric switching devices are called valves as both circuits on nomenclature. The hydraulic switching control on huge working power have been disclose only a few years ago, at first in the world on JFPS Spring Meeting on Fluid Power", May 2002 in Japan. This paper is described process of some experiment to have evidence to practical use of the Hydraulic Switching Control. We have obtained patents about the hydraulic switching control as a new theory on the Fluid Power Systems, and registered it as the trade mark "FST" means Fluid Switching Transmission. Outline of the performance of the FST is also described in this paper.
\end{abstract}

\section{KEY WORDS}

Key words, Hydraulic switching, Electronic switching, Fluid switching Transmission, FST

\section{NOMENCLATURE}

$Q m:$ output torque of motor

$Q p:$ input torque of hydraulic pump

$I$ : inertia of flywheel (sum of inertia on motor shaft)

$\omega:$ angular velocity of flywheel

$a:$ on/off condition of un-loader valve

$l$ : current of electric power sauce

Es 1: output voltage of electric power sauce

Ec1 : output current of electric power sauce

L1: inductance of reactor cil

$b:$ on/off condition of switching device

\section{INTRODUCTION}

The concept of hydraulic switching for power control and regulate the fluid conditions on the hydraulic power system was have not existed in the world, it is as same as a seen on electronics a half century ago. It was believed that the power switching on high frequency on heavy load, it was believed that makes severe vibration as parasite oscillations.

We have succeeded to suppress the violence of transient in the power electronics, based on the way of the electron beam deflection and fly back circuit on CRT control, especially for color CRT display.

Usually, human lives in the world that is dominated by the ILLUSION. However on the physical object like as the mechanical constructions, the vibration makes serious obstructions, it is much different from the human sense and sensitivity of human. 
The technology of FST (Fluid Switching Transmission : is only our registered trademark) is facing the difficulties on vibrations not only physical but also illusion of human sensitivities.

The switching technology on the hydraulic power system will be judged from the quite otherwise. We have succeeded on a trial of the production of hydraulic power switching servo controllers reciprocate, it has brought the great successful result of the trial for us.

We continued the development of the hydraulic power control by switching since 1973, accompany with the research on the electronic switching power control, to increase their transferring efficiency on the power transmission and suppression of unpardonable obstructions will be eliminated.

We have disclosed the hydraulic switching technologies on May 2002, applied for a patent simultaneously, and we have obtained the patent of the theory on hydraulic switching technology recently, what is said ${ }^{(1)(2)(3)(4)(5)(6)}$.

We will discuss about the technology of power switching on the hydraulic and electric field of technology, and investigate the analogy of the hydraulic switching technology with the electronic switching power control. The prototype of rail vehicles will be introduced in this paper which are produced by us, in order to prove the performance of the hydraulic switching technology as a transmission, both to hydraulic and electric switching power control systems. The three units of original servo systems are shown in Figure 1, they are a part of prototype produced at first, controlled by hydraulic power switching. These servo actuators are produced 1973 in order to cut down the energy consumption of a hydraulic servo systems working in a factory as productive facilities, and the FST servo control system succeeded to save the energy more than $99 \%$ of conventional servo control systems in that was running in the factory.

These original models have mechanical output of reciprocate.

The servo control system has an excellent ability as power transmission if designed appropriately.

We have produced plot type of LRV (Light Rail Vehicle) to prove the performance of power switching control systems as the transmission of vehicles on board.

Figure 2 shows the plot type of trolley less electric vehicle powered by the EDLC (Electric Double Layer Capacitor) on board, the vehicle is driven by electrostatic energy at first in the world, $t$ is shown in Figure 2 has electronic power switching controllers as transmission, for powering and regenerative brake.

Figure 3 is the vehicle powered by the thermal engine and flywheel hybrid, and the power is controlled by the bilateral FST $^{(7)(8)(9)(10)}$.

The vehicle shown in Figure 3 is powered by the kinetic energy stored in the flywheel on board, and on braking, kinetic energy that was kept in vehicle returns to the flywheel as regenerative brake system.

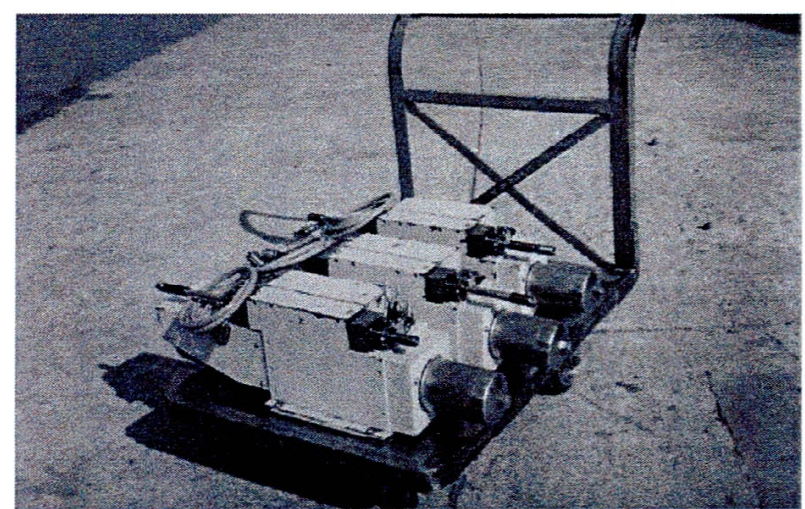

Figure 1 Switching servo control produced 1973

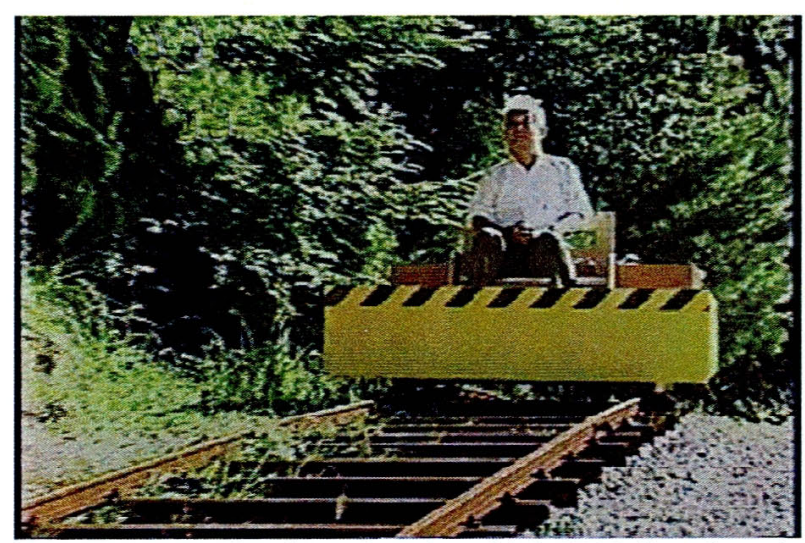

Figure 2 Trolley less electric vehicle EDLC on board

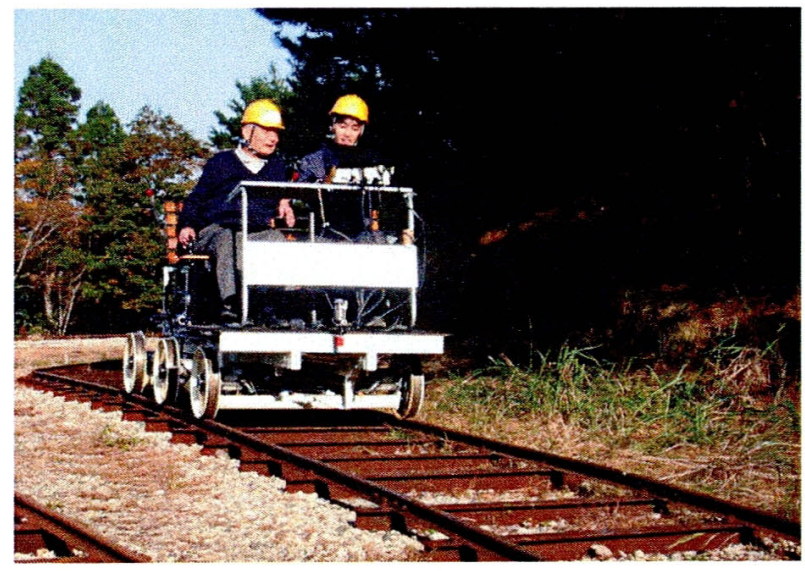

Figure 3 Hybrid flywheel vehicle FST on board

The FST is a high performance transmission, and the FST vehicle has the powering system equivalent to that of the electric systems.

FST have been proved that its operating efficiency is $100 \%$ on theoretically, same to that of the electronic switching power control designed proper.

The analogy between hydraulic system and that of electricity is often appears on the books telling technical theory, however almost of them are mistake of slip.

The excellent power transmission will perform energy regeneration on the powering system, and regeneration 
demands a support of accumulators of high performance that will be introduced bellow

\section{THE POWER SWITCING}

Generally importance of the power switching on the power systems have been well known on the field of technology, the electronic switching technology have only grow to a standard system of power controllers.

However we have completed hydraulic power switching control system more than 30 years ago as servo controllers, however, we did not disclosed them.

The most basic hydraulic switching circuit diagram of the "FST" is shown Figure 4.

Figure 5 shows the electronic circuit diagram equivalent with the FST circuit what is shown in Figure 4.

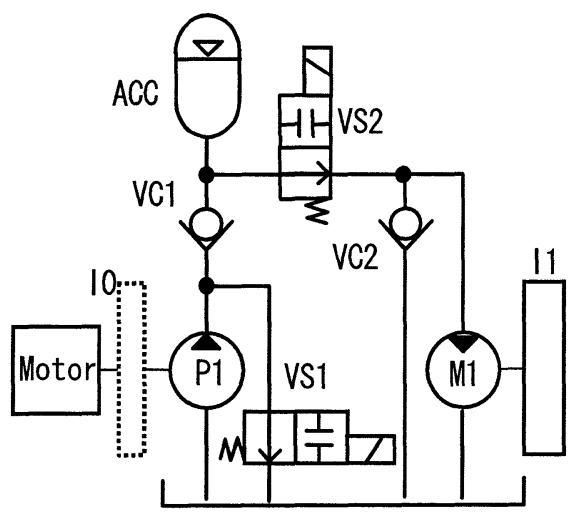

Figure 4 Circuit diagram of the FST

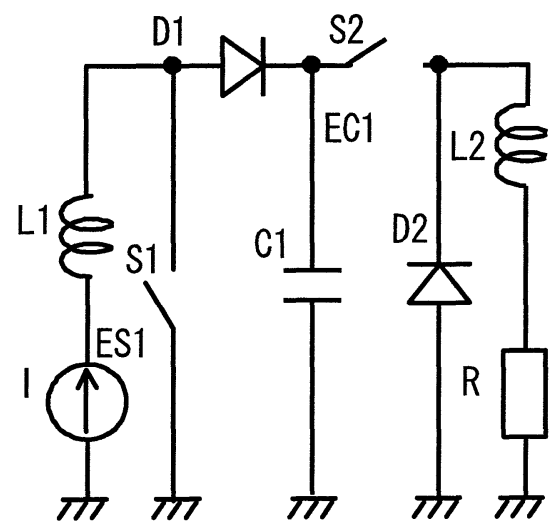

Figure 5 Circuit diagram of a switching converter

Figure 6 to Figure 9 are supplementary explain about the principles of FST.

Function of VS1 in Figure 4 behaves like as the un-loader valve, but its usual operating frequency is much higher than generally used un-loader valves.

It is operated synchronize to clock frequency or self oscillating frequency that is decided by the way of

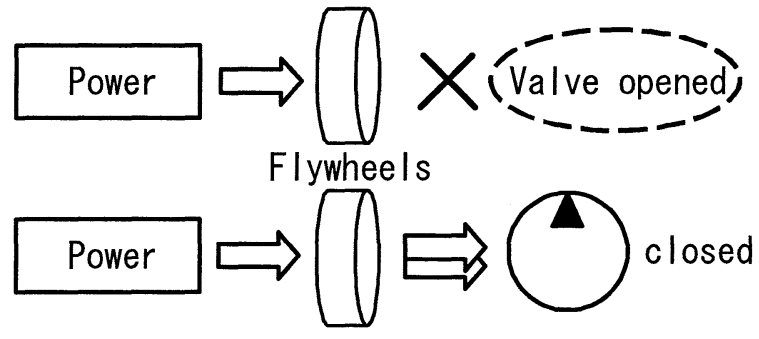

Figure 6 Inertia makes two phases

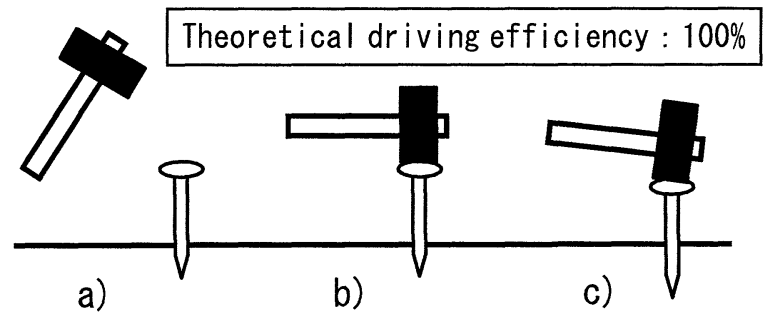

Figure 7 Drive a nail

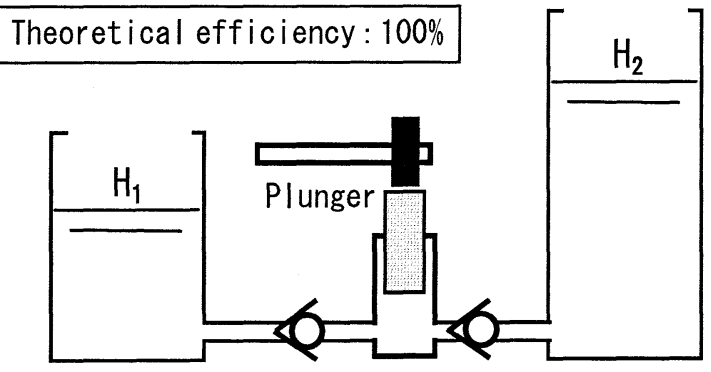

Figure 8 Drive a plunger pump by hammer

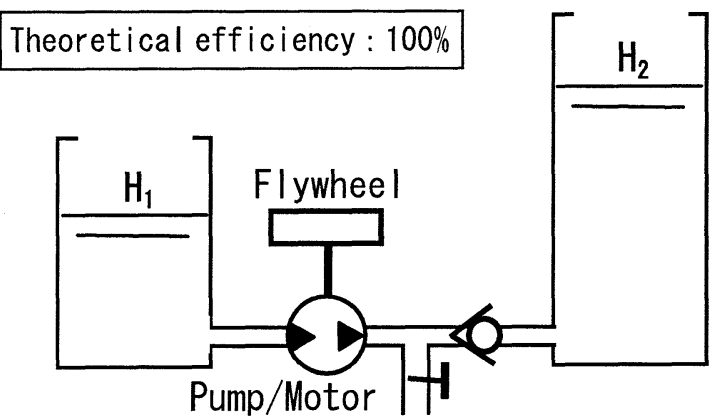

Figure 9 Inertia makes boost the hydraulic pressure

feedback loop from the hydraulic pressure in the accumulator ACC 4 in Figure 4.

Figure 6 shows the behavior of torque transfer, when the un-loader valve is switched, on the rotating motor shaft installed hydraulic pump and flywheel or incidental inertia instead of flywheel.

When the un-loader valve is opened, the angular velocity of flywheel increased by the motor torque. 
The un-loader valve is closed the hydraulic pump will be driven by larger torque than motor torque, sum of the torque of motor and flywheel.

Some of similar examples of switching action were described before, on the analytical explanation.

Figure 7 shows a process of driving a nail into a wooden block. The kinetic energy of hammer is converted into a thrust to drive a nail, and the efficiency on driving nail is $100 \%$, if the coefficient of elasticity, " $E$ " $=1$, between the head of hammer and the nail, theoretically.

An example similar to the nail drive is shown in Figure 8. A plunger works as a ram, that is driven by a hammer, then, they make a piston pump, and boost up the hydraulic pressure $\mathrm{H} 1$ to $\mathrm{H} 2$.

Theoretical efficiency of this plunger pump also presents $100 \%$ on the pressure boost.

An improved pressure booster is shown in Figure 9, equivalent to the boosters in Figure 7 and 8.

This booster system works as rotary machine, generally, the rotary machines are superior to reciprocate for practical use, especially its continuative transfer ability is excellent, and the power density of rotary machines are better than that of reciprocate.

An example of switching control hydraulic power system will be analyzed on the equations.

$Q m$ : torque, motor generates, $Q p$ : loaded torque of hydraulic pump, $I$ : sum of inertia on the pump driving shaft, $\omega$ : angular velocity of the driving shaft.

The motion is shown by Eq. (1).

$$
\begin{aligned}
& Q m-a Q p-I d \omega / d t=0 \\
& a=1: \text { VS1 is closed } \\
& a=0: \text { VS1 is opend }
\end{aligned}
$$

On the electric circuit that equivalent with hydraulic switching circuit, the voltage boost is practiced as the followings; $i$ : sauce current, ES1: voltage of sauce, $L 1$ : inductance of $L 1, E C 1$ : output voltage on the terminal of C1.

The relation between current and voltage is shown by Eq. (2), it is equivalent with Eq. (1).

$$
E S 1-b E C 1-L 1 d i / d t=0
$$

$$
\begin{aligned}
& b=1: \mathrm{S} 1 \text { is opend (OFF) } \\
& b=0: \mathrm{S} 1 \text { is closed (ON) }
\end{aligned}
$$

There are various way to present the power switching both to mechanical and electric engineering field.

Then, we will present a typical response on switching both to hydraulic and electronic switching.

Figure 10 shows the angular velocity of flywheel connected with hydraulic pump and motor coaxially, and the magnetic flux density in the core of inductor on

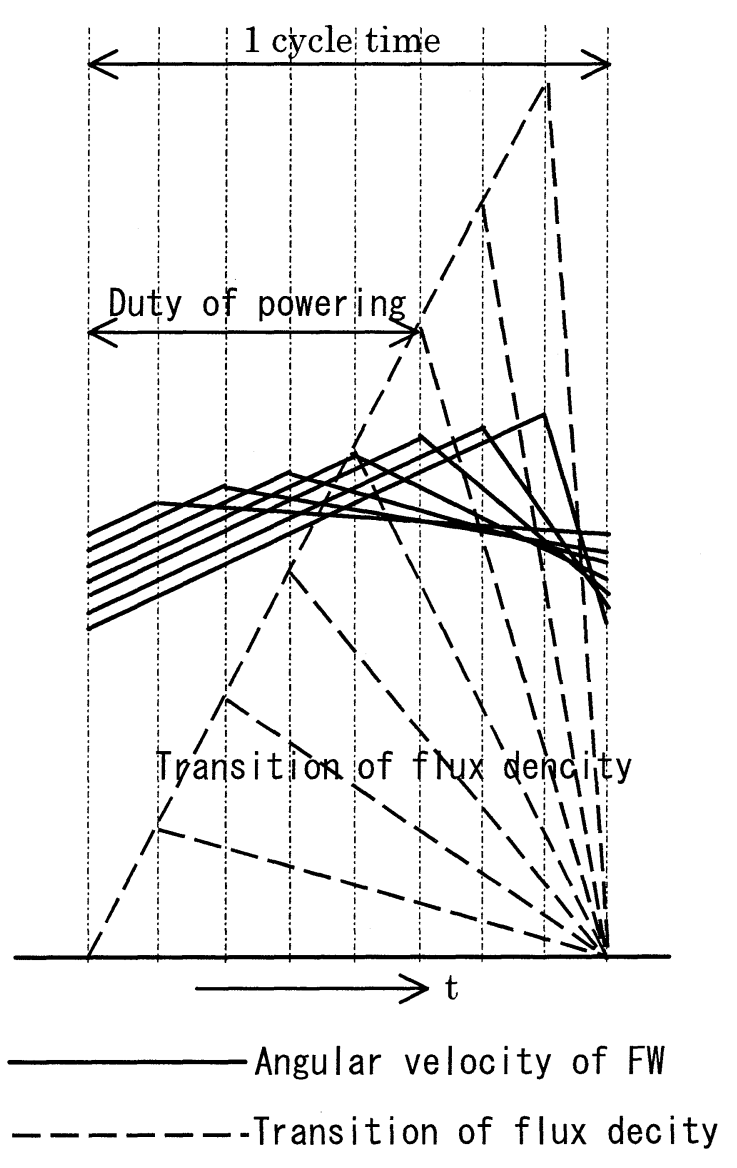

Figure 10 Transition of angular velocity and flux

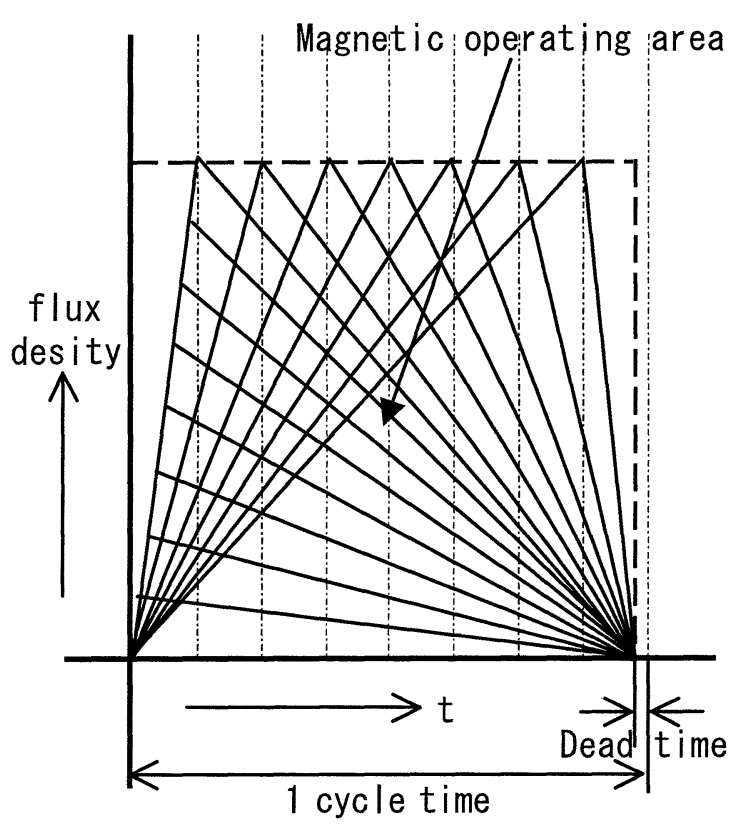

Figure 11 Magnetic flux density on 2nd quadrant 
a pressure and a voltage booster..

The transition of magnetic flux density in the inductor core on chopper converter is shown in Figure 11, generally magnetic cores of inductor are operated on the 1 st quadrant and 3rd quadrant of the B-H plain, and operating area is limited by characteristics of magnetic core.

The hydraulic switching system is equivalent with electronic switching system, but it is not equal on all, there are various inconsistency between on both system. Generally the switching power controls are applied pulse width modulation (PWM).

There are various method to apply PWM for switching, but they are belongs to the system of synchronous to clock, or hysteresis control that has no clock pulse.

You can chose whichever you like above two ways of PWM control, but if anything asynchronous hystersis control is better than others, limited on hydraulic system.

Anyway the switching power control is constructed of reciprocal transitions of the energy accompany with oscillation

\section{APPLICATIONS}

We have produced many instruments to prove the performance of switching power control not only hydraulic power systems but also on the electric power control systems.

Figure 12 shows the Flywheel Hybrid Rail Vehicle controlled by the hydraulic switching transmission on left hand, and on right the Electric Rail Vehicle powered by the electrostatic energy stored in the Electric Double Layer Capacitor (EDLC) on board. ${ }^{(8)(9)(10)}$

Close up of the flywheel rail vehicle is shown in figure 13 , the instrument panel and bench seat for operator is seamed on the head of the vehicle.

Middle of the vehicle, under the seat, flywheel is mounted, reserve tank and the circuit hydraulic switching transmission are on near the tail of vehicle, accumulators are also mounted top of the hydraulic control circuit.

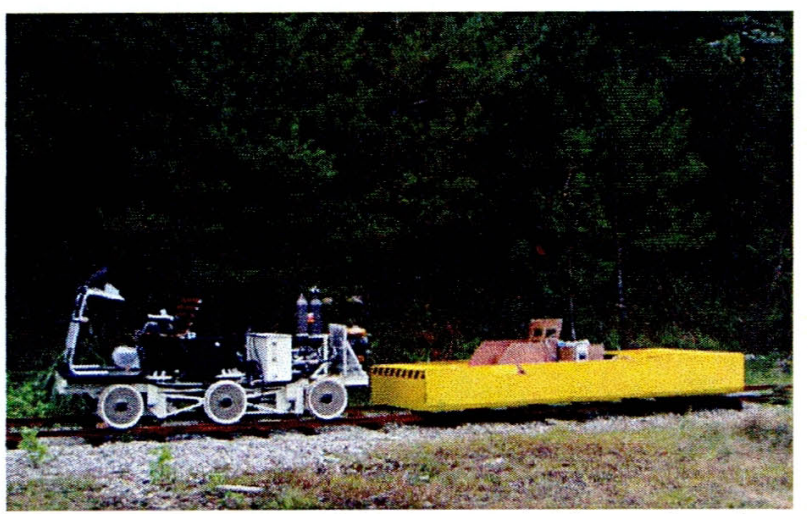

Figure 12 Rail vehicles have switching on board

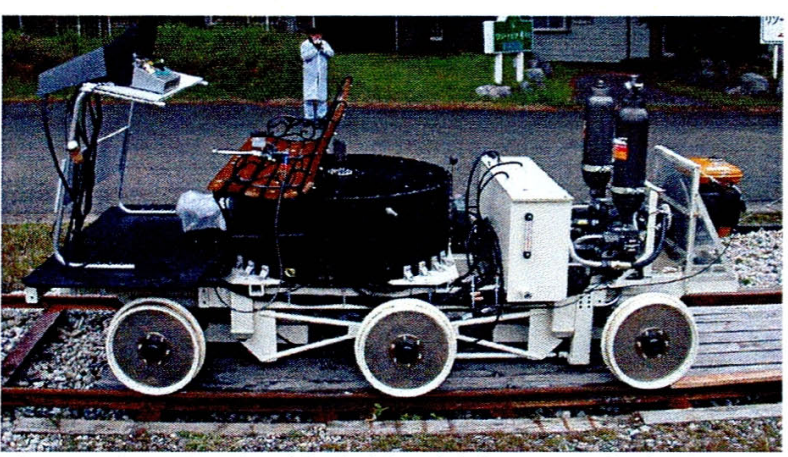

Figure 13 Flywheel hybrid vehicle controlled FST

The thermal engine is mounted on tail end of the vehicle it is shown in Figure 13.

The vehicle runs powered by the flywheel on board which is increased its angular velocity by the engine power and regenerative braking power.

The vehicle on the right hand in Figure 12 is the prototype of rail vehicle powered by EDLC on board.

The variable range of FST-transmission is boundless and the efficiency as transmission have been measured over $99 \%$ on wide range of convert ratio.

\section{POWER ACCUVULATORS}

It is important for us that reduce energy consumption of vehicles and other instruments, so we continued to develop of high efficient motor, transmission and power accumulator.

The high efficient vehicles must be equipped regenerating brake and high efficient bilateral transmission onboard.

We have established switching power transmissions on electronic and hydraulic power control, and we found the necessity of power accumulators not only on hydraulic bat electric power switching systems.

We made a trial to develop the power accumulators since 1975.

One of successful power accumulator is the EDLC and other is the Flywheel covered with floating shrouds.

Newly developed flywheel as power accumulators has floating shrouds rotating coaxially with flywheel, the flywheel able to rotate up to super sonic velocity on its tangential velocity in the atmospheric air pressure.

The flywheel is shown in Figure 15, this is mounted on board FST vehicle, it wears three layer of shrouds, in Figure 13, upper half of shrouds are removed for exhibition.

Figure 16 shows a cross section of the flywheel.

The aerodynamic theory of reducing drag force of the flywheel in atmospheric air pressure environment is our patent.

Figure 17 shows EDLC which has huge electro static capacity of 2,000 and 1,200 Farad, we have continued development of powering by the EDLC, and our first EDLC vehicle was succeeded 1988. 


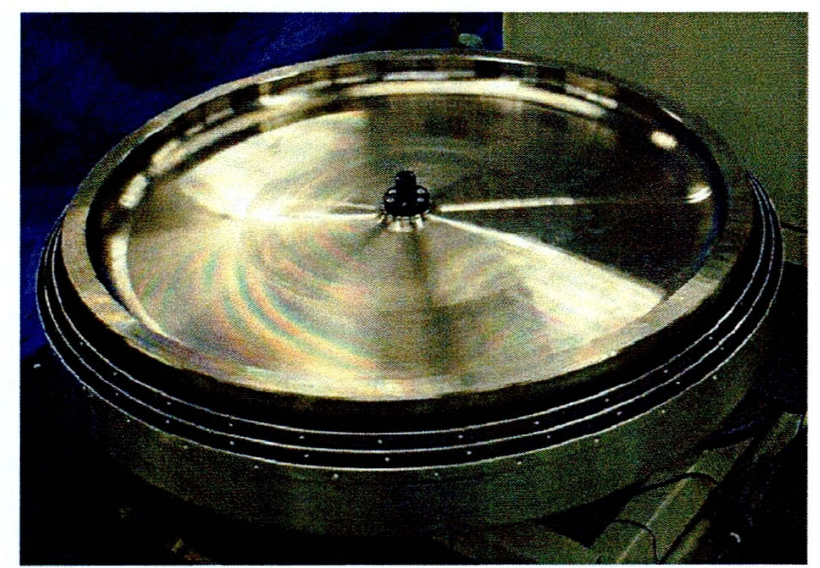

Figure 15 Flywheel with bottom shroud

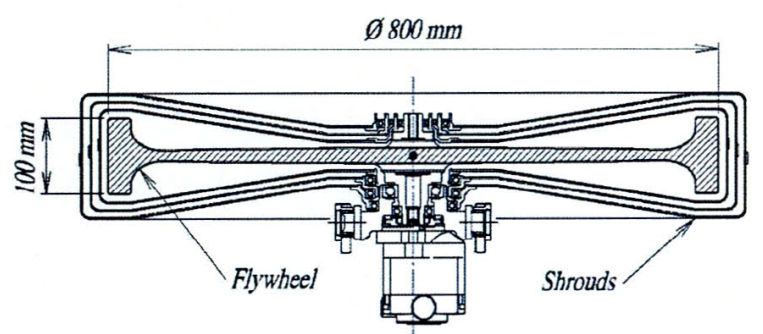

Figure 16 Cross section of flywheel and shroud

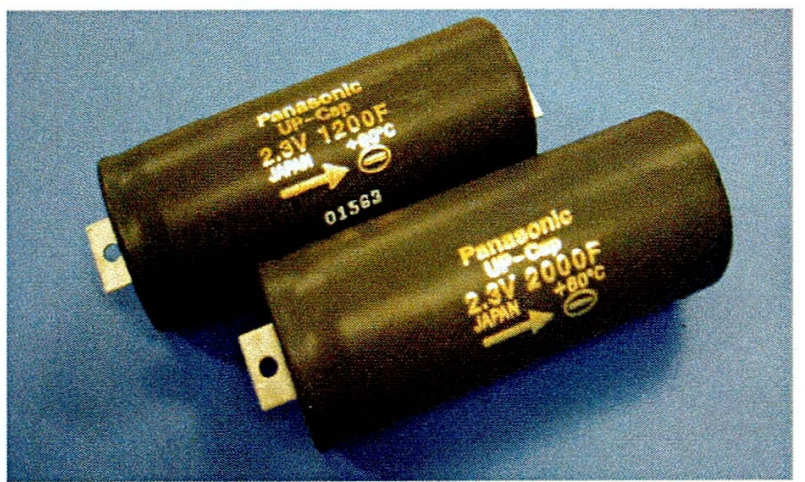

Figure 17 Electric Double Layer Capacitor

Now, we are converting a conventional marine Diesel engine into the FST control engine, in order to present the effect of FST on Diesel engines.

The FST involves the concept of dynamics, on the rotary inertia it makes transmission, on the reciprocate, FST makes most efficient hydraulic system.

The FST engine will complete in September 2005, and it will begin the test run.

The full detail of the experiment is will be disclosed near future.

We have chose 4-stroke cycle Diesel engine in this project in order to prove the performance of FST.

The difficulties applying to 4 -stroke will be solved by the FST engine control systems.

\section{CONCLUSION}

We proved that the FST is one of best way to keep the efficiency of transmissions, thermal engine and other powering systems. The best way to reduce consumption of energy is the increase of efficiency on systems, we believe.

The power switching is completed on the electric power control technology, but it was not exist on the hydraulic power system.

We succeeded to make practicable hydraulic power switching at first in the world, so we cannot find any publish about hydraulic power switching yet.

\section{REFERECE}

(1) The 8 th National Symposium on Power and Energy Systems (SPES 2002)

Itoh, Seki, Aoyama and Shimada, Flywheel for energy storage, p365-368, June 18 2002, Tokyo.

(2) Itoh, Seki, Aoyama, Shimada and Suzuki, The new theory and technology on hydraulic transmission, JFPS Spring meeting 2002, p34-39, May 23 2002, Tokyo,

(3) Seki and Itoh, An Examination of a LRV and its Transmission equipped onboard, p91-93,

Itoh and Seki, The equivalent Hydraulic Transmission on Electric Transmission, p94-96,

Itoh and Seki, The JFPS Spring meeting 2004, p34-39, May 26 2004, Tokyo.

(4) Itoh, Trolley less vehicle and energy accumulator, Rolling Stock \& Technology, No89, September 2002, Rail \& Tech. Publishing Co.Ltd..,

(5) Itoh, The proposal on hydraulic control technology, Hydraulic \& Pneumatics, Vol.42 No.5, p49-55, 2003

(6) Itoh, Switching control on the hydraulic power system, Hydraulic \& Pneumatics, Vol.42 No.7, p55-60, 2003

(7) Itoh, An application of shrouded flywheel, Hydraulic \& Pneumatics, Vol.43 No.12, p4-10, Oct. 2004, Nippon kogyoshuppan.

(8) Itoh and Ski, We produce a LRV and constructed a railroad for experiment, S4-2-6 p265-268, J-RAIL-01, December 12 2002, tokyou

(9) Seki and Itoh, Application of Electric Double Layer Capacitor for LRV and EV, S4-1-2, p329-332, J-RAIL2002, November 27 2002, Kobe

(10) Itoh and Seki, Light-Rail-Vehicle running only by the storage energy of on board EDLC , and steering by the Automatic Control Links, 3302, P247-250, TRANSLOG2002, December 11 2002, Kawasaki 